\title{
The design and assessment of the therapeutic potential of apelin-13 peptide mimetics for alleviating metabolic dysfunction in diabetes and obesity
}

\author{
Finbarr O’Harte \\ Ulster University, United Kingdom
}

https://doi.org/10.17952/35EPS.2018.010

\section{Introduction}

To combat Type 2 diabetes mellitus (T2DM) there is an urgent need for development of new multi-faceted pharmaceutical agents, which induce weight loss and decrease both hyperglycaemia and associated complications, without causing adverse effects. Apelin is a circulating adipokine produced and secreted mainly by adipocytes and endothelial cells [1]. Several bioactive isoforms of apelin exist, including apelin-12, -13, -16, -17, -19 and -36 [2]. The apelin receptor, APJ is present in most tissues and the apelin/APJ (apelinergic system) has been shown to be involved in multiple metabolic processes including control of glucose homeostasis [3]. Rapid degradation and short half-life of native apelin isoforms $(4-7 \mathrm{~min})$ severely hinders the pharmacological exploitation of apelin peptides. To overcome the short half-life of apelin-13 related peptides, we have developed a range of enzyme resistant analogues. Two of these acylated analogues include (Lys ${ }^{8} \mathrm{GluPAL}$ )apelin-13 amide and pGlu(Lys $\left.{ }^{8} \mathrm{GluPAL}\right)$ apelin-13 amide [4]. These stable apelin analogues stimulated insulin secretion from clonal pancreatic beta cells, primary culture of isolated mouse islets cells and proved to be the most potent of a series of analogues studied [5]. In the present study, metabolic and weight reducing effects of chronic (28 day) once daily administration of (Lys $\left.{ }^{8} \mathrm{GluPAL}\right)$ apelin-13 amide and pGlu(Lys $\left.{ }^{8} \mathrm{GluPAL}\right)$ apelin-13 amide were directly compared to the well-established acylated GLP-1 receptor mimetic liraglutide, using a high-fat fed mouse model diet-induced obesity-diabetes (DIO).

\section{Methods}

Apelin analogues were synthesised by EZ Biolabs (Carmel, IN, USA) at $>95 \%$ purity. A gamma-glutamyl spacer (also found in liraglutide) with palmitate adjunct (GluPAL) was added to the side-chain of apelin Lys ${ }^{8}$ to promote binding to plasma proteins and reduce renal clearance, thus extending its in vivo bioactivity. Male NIH Swiss mice (Harlan UK Ltd., Blackthorne, UK) were maintained on a high fat diet (45\% fat, Dietex International Ltd., Witham, UK) from 8 weeks old for a total of 150 days to evoke insulin resistance and diet-induced obesitydiabetes (DIO). Control mice was maintained on standard rodent diet (10\% fat, Trouw Nutrition, Cheshire, UK). Groups of normal control and high-fat fed (HFF) mice $(n=8)$ received once daily ip injections of either $0.9 \%$ saline vehicle (lean and high fat controls) or (Lys ${ }^{8} \mathrm{GluPAL}$ )apelin-13 amide, pGlu(Lys ${ }^{8} \mathrm{GluPAL}$ )apelin-13 amide or liraglutide (each at $25 \mathrm{nmol} / \mathrm{kg} \mathrm{bw}$ ) over a 28 day treatment period. The various once daily peptide treatments were extended beyond this period (up to 40 days) to allow for additional post-treatment investigations to be performed. Food intake, bodyweight, non-fasting blood glucose and plasma insulin concentrations were measured every 2-3 days up to day 28 . Following the 28 day period, $16 \mathrm{~h}$ fasted mice were tested for ip and oral glucose tolerance, as well as an insulin sensitivity ( $25 \mathrm{U} / \mathrm{kg}$ insulin challenge). Blood samples were collected from tail snips of mice and blood glucose (Ascencia Contour meter) and HbAlc (PTS Diagnostic, IN, USA) were measured. Blood was taken from fasted mice for measurement of lipid profiles including HDL-cholesterol, LDL-cholesterol and triglyceride levels by an ILab 650 Clinical Analyser.

\section{Results}

Both acylated apelin-13 amide analogues significantly decreased non-fasted blood glucose $(\mathrm{P}<0.05$ and $\mathrm{P}<0.01$ Anova; Fig 1) and increased non-fasting plasma insulin (data not shown). Cumulative energy intake was also significantly decreased in apelin and liraglutide treated mice $(27 \%$ decrease, $\mathrm{P}<0.01$; Fig. 1$)$. A significant decrease in bodyweight was noted over 28 days (3-7\% bodyweight reduction), with all treatment groups compared to lean and high-fat fed saline treated mice $(\mathrm{p}<0.01$ and $\mathrm{p}<0.001 ;$ Fig. 1). Apelin analogues and liraglutide showed an improved ip glucose tolerance (Fig. 1), reduced glycated haemoglobin (HbA1c Fig. 1) and improved insulin sensitivity (Fig. 1). All peptides showed a reduction in circulating triglycerides (Fig. 1G) and 


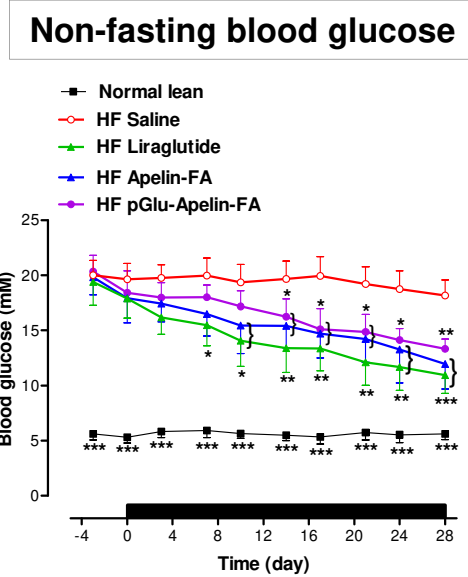

\section{Cumulative energy intake}
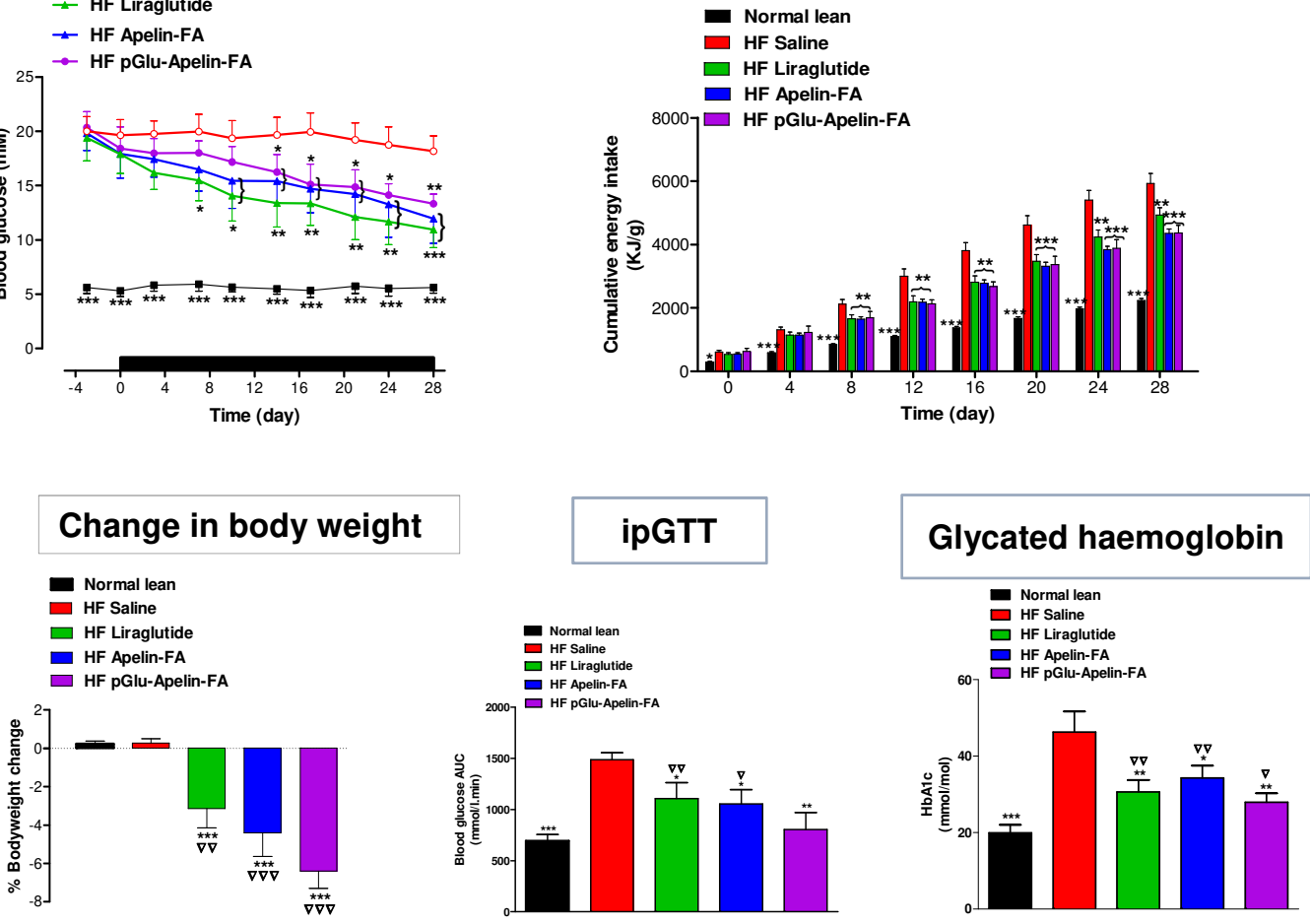

Insulin sensitivity

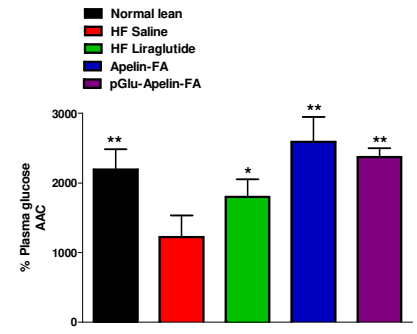

\section{Triglycerides}
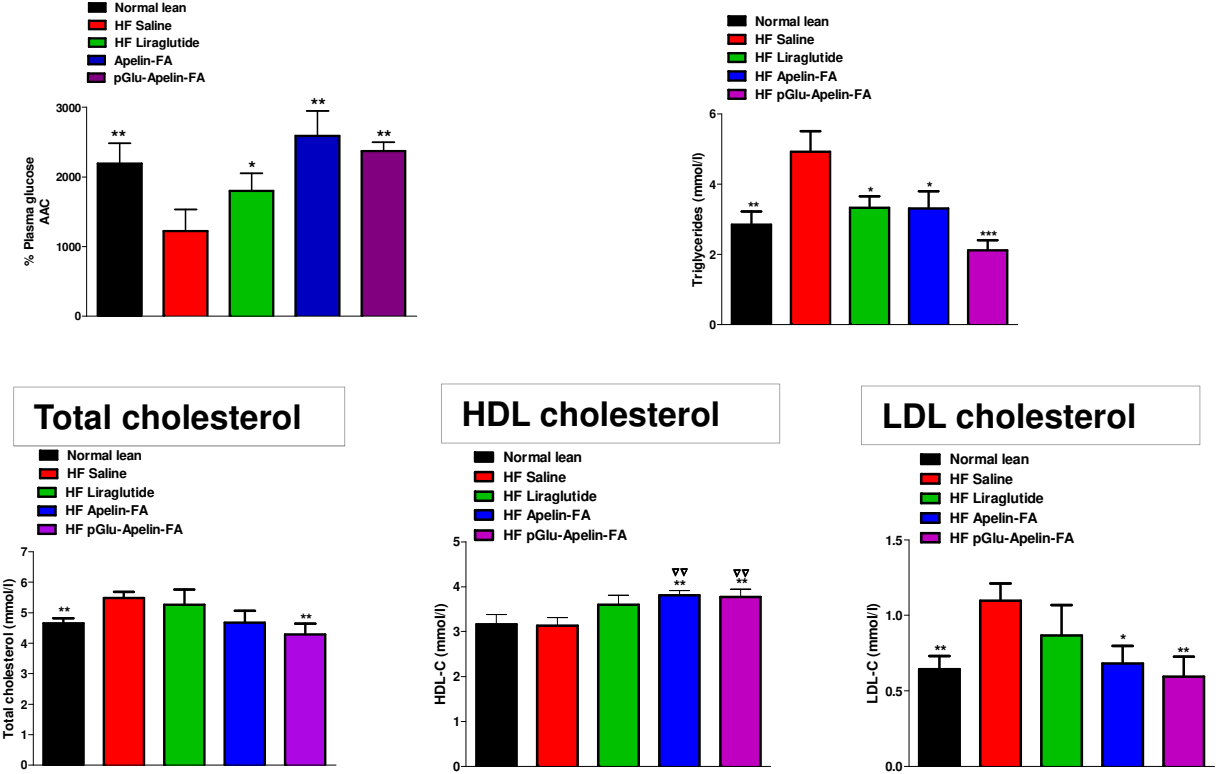

Figure 1 
both acylated apelin analogues demonstrated an improved lipid profile for HDL and LDL cholesterol compared to liraglutide (Fig. 1).

\section{Discussion}

Recent studies indicate an emerging involvement of apelin in energy metabolism and the pathophysiology of obesity [6]. In the present study, once daily (Lys ${ }^{8}$ gluPAL)apelin-13 amide and pGlu(Lys ${ }^{8}$ gluPAL)apelin-13 amide were compared to liraglutide in DIO mice in a 28 day study. Acylated apelin analogues resulted in marked reductions in energy intake as well body weight, improvements in non-fasting glucose, reduced glucose excursion after an ip glucose tolerance test and significantly reduced levels of glycated haemoglobin (HbA1c). The improvements may also reflect improvement in insulin action as evidenced by enhanced hypoglycaem ic action of exogenous insulin. Apelin and its APJ receptors have been detected in the arcuate and paraventricular nuclei of hypothalamus, known to be key sites in central control of feeding behaviour and energy expenditure $[7,8]$. Apelin could also alter body adiposity independent of food intake by increasing energy expenditure [9]. Interestingly, apelin knockout (KO) mice exhibit reduced insulin sensitivity, glucose intolerance and hyperinsulinaemia [10]. In the present study with high fat fed DIO mice, pGlu(Lys ${ }^{8}$ gluPAL)apelin-13 amide reduced total cholesterol and both acylated apelin analogues reduced circulating triglycerides, LDL-cholesterol as well as increasing HDL-cholesterol. Cardiovascular benefits of apelin, including a reduction of blood pressure are already established [11]. Once daily administration of the GLP-1 mimetic, liraglutide replicated all of the benefits of apelin-13 analogues but it failed to completely improve lipid profile in DIO mice as shown previously in our laboratory [12]. In conclusion, the present study has shown that once daily administration of (Lys ${ }^{8}$ gluPAL)apelin-13 amide or pGlu(Lys ${ }^{8}$ gluPAL)apelin-13 amide ameliorated diabetes, evoked weight loss and decreased circulating lipids in DIO mice, with effects equivalent to or better than liraglutide. Overall the pGlu(Lys ${ }^{8}$ gluPAL)apelin-13 amide was the most effective analogue and was better than it equivalent nonacylated analogue tested previously [7].

\section{References}

1. Boucher J, Masri B, Daviaud D, Gesta S, Guigne C, Mazzucotelli A et al. Endocrinology 2005; 146:17641771.

2. Kawamata Y, Habata Y, Fukusumi S, Hosoya M, Fujii R, HinumaS, et al. Biophysica Acta 2001; 23:162-171.

3. O’Carroll AM, Lolait SJ, Harris LE, Pope GR. J Endocrinol. 2013; 219:R13-R35.

4. O’Harte FPM, Parthsarathy V, Hogg C, Flatt PR. Biochem Pharmacol. 2017;146: 165-173.

5. O'Harte FPM, Parthsarathy V, Hogg C, Flatt PR. Peptides. 2018;100: 219-228.

6. Bertrand C, Valet P, Castan-Laurell I. Front Physiol. 2015; 6:115.

7. Parthsarathy V, Hogg C, O'Harte FPM, Flatt PR. Diabetes Obes Metabolism. 2018; 20:319-327.

8. Valle A, Hoggard N, Adams AC, Roca P, Speakman JR. J Neuroendocrinol. 2008; 20:79-84.

9. Higuchi K, Masaki T, Gotoh K, Chiba S, Katsuragi I, Tanaka K, et al. Endocrinology. 2007; 148:2690-2697.

10. Yue P, Jin H, Aillaud M, Deng AC, Azuma J, Asagami T, et al. Am J Physiol Endocrinol Metab. 2010; 298:59-67.

11. Mughal A, O’Rourke ST. Pharmacol Ther. 2018;pii: S0163-7258(18):30093-30097.

12. Millar P, Pathak N, Parthsarathy V, Bjourson AJ, O’Kane M, Pathak V et al. J Endocrinol. 2017; 234:255-267.

\section{Acknowledgements}

We would like to thank the funders DfE for awarding a PhD studentship to $\mathrm{CH}$. Also, we thank Invest NI for their Proof of Concept PoC518 grant funding. 